\title{
THE IMPACT OF GLOBAL ECONOMIC POLICY UNCERTAINTY AND VOLATILITY ON STOCK MARKETS: EVIDENCE FROM ISLAMIC COUNTRIES
}

\author{
Norashikin \\ Adam $^{1+}$ \\ (iD) Noor Zahirah \\ Mohd Sidek ${ }^{2}$ \\ Arshian Sharif ${ }^{3}$
}

\author{
${ }^{1,2}$ Faculty of Business Management, Universiti Teknologi MARA, Malaysia. \\ Email:norashikinadam@uitm.edu.my Tel: +6012-6498131 \\ 'Email:nzahirah@uitm.edu.my Tel: +6013-4839896 \\ ${ }^{3}$ Othman Yeop Abdullah Graduate School of Business, Universiti Utara \\ Malaysia, Malaysia. \\ ${ }^{3}$ Email:arshian.aslam@gmail.com Tel: +923333274789
}

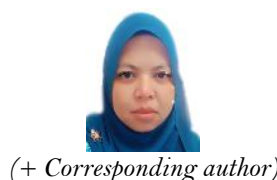

(+ Corresponding author)

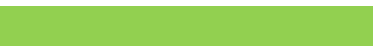

Article History

Received: 1 November 2021 Revised: 6 December 2021

Accepted: 20 December 202

Published: 17 January 2022

\section{Keywords}

Islamic stock market Global economic policy Uncertainty

Volatility index

Wavelet

COVID-19.

JEL Classification: C22, G15.

\section{ABSTRACT}

This study investigates the impact of uncertainty and volatility on ten Islamic stock returns using monthly data for from 2011:M5 to 2021:M5. We rely on the continuous wavelet transform and wavelet coherence ratios, which allows decomposition of time series across time scales to investigate the causal relationship between stock market returns, economic policy uncertainty and volatility. Our results provide some interesting insights. First, economic policy uncertainty, in general, has a negative effect on the majority of Islamic stock returns, except for the Dow Jones Islamic Market (DJIM). Second, volatility has a significant positive impact on most of the Islamic stock returns in various countries. Third, both economic policy uncertainty and volatility have a greater impact on the Islamic stock returns post-COVID-19 outbreak. These results should assist investors to re-evaluate their portfolios to fully maximize the potentials of these Islamic stock markets. Policymakers could use these results to design policies to reduce economic policy uncertainty as well as to cushion the impact of externally generated uncertainties.

Contribution/Originality: The paper's primary contribution is to assess the impact of uncertainty on the Islamic stock market index. These findings could help investors re-evaluate their portfolios to fully exploit the potentials of the Islamic stock markets. These findings could be used by policymakers to develop measures that reduce economic policy uncertainty while also mitigating the impact of externally created uncertainties.

\section{INTRODUCTION}

Islamic stocks trade on the same exchange as traditional stocks, although the former must meet certain Shariah requirements. Nonetheless, their performance varies according to their location, product type, crisis connection, size, and efficiency. Given these factors, investors focus on areas where they may get higher returns through international diversification and share risk within the markets. The rise of Islamic stocks in developing economies has highlighted other areas where competition might increase system efficiency. The penetration of Islamic stocks into the developed market, where transactions are more efficient than in emerging markets, is a noteworthy trend. It's debatable if Islamic stocks performed better during the financial crisis, and although the Islamic financial industry is expanding at an unprecedented rate in developing and emerging nations, the outward appearance of their results does not guarantee comparable results in other places. 
The stock market has had several ups and downs over the years. Many studies have been conducted to investigate the reasons that contribute to these changes, including macro and microeconomic factors, oil price movements, inflation, recession, and interest rate movements, among others. We focus on economic policy uncertainty and volatility in this study since the elements that contribute to the stock market's ups and downs can be explained by economic policy uncertainty or volatility. The COVID-19 pandemic, for example, has affected lives in all countries and communities, and had a negative impact on global economic growth in 2020 unlike anything seen in over a century. As factories closed and lockdown measures extended around the globe, restricting mobility and commercial operations, businesses are dealing with lost revenue and interrupted supply chain networks. In addition, unemployment is at an all-time high. Despite the fact that authorities throughout the world are racing to put fiscal and monetary measures in place to mitigate the effects of the recession, uncertainty remains high as countries endure multiple waves of COVID-19 infections. All of this generates a sense of ambiguity as volatility is predicted to rise in lockstep as uncertainty rises.

In empirical investigations, economic policy uncertainty has been found to impair economic performance. Uncertainty has a direct impact on stock market participation and performance through influencing economic agents' decisions about employment, consumption, saving, and investing (Arouri, Estay, Rault, \& Roubaud, 2016; Gulen \& Ion, 2015). Economic policy uncertainty has an impact on the financing and investment climate, which, in turn, has an impact on stock market performance due to lower involvement from domestic and international investors. Furthermore, economic policy uncertainty has an impact on commodity markets, such as oil prices, which, in turn, has an impact on the stock market and economic results (Kang, Gracia, \& Ratti, 2017b; Kang, Ratti, \& Vespignani, 2017a; Kang \& Ratti, 2013).

The Islamic stock market offers an alternative to investors looking for markets to diversify their portfolios. However, more empirical research into how these markets react is needed to better understand whether their behavior is similar to that of their traditional counterparts. As a result, this article adds to our understanding of the impact of global economic policy uncertainty (GEPU) and the volatility (VIX) on investment and asset pricing decisions for investors.

This paper offers contributions from the following viewpoints. To begin, most empirical research has measured the impact of economic policy uncertainty on stock market returns by looking at economic policy uncertainty in developed economies. Therefore, this study aims to fill a knowledge gap in the literature by bringing new information on how economic policy uncertainty affects Islamic stock returns.

Second, to capture the time-varying nonlinear impact of GEPU and the VIX on stock market returns, this study employs the Wavelet Coherence approach. We use the continuous wavelet transform (CWT) and wavelet coherence (WC) analysis. The wavelet transform can provide us with the frequency of signals as well as the time associated with those frequencies, which makes it particularly useful in a variety of fields. Multiple structures (equations) are used in this model to characterize the behavior of time series over various time scales. By permitting the time and period between these structures, this method can capture more complex and dynamic patterns.

Third, this study focuses on the Islamic stock market, which is relatively underexplored, and it would provide an interesting perspective. Hence, this study extends the existing literature by adding new evidence relating to how uncertainty affects the Islamic stock market returns. Results should provide directions for investors to formulate better investment strategies and asset allocation. The rest of the paper is organized as follows: Section 2 reviews a selection of literature related to this study; Section 3 describes the data and methodology used in this study; Section 4 discusses the empirical results and findings; and the final section concludes.

\section{REVIEW OF SELECTED LITERATURE}

Due to its impact on investors' profitability, portfolio flows, stock market liquidity, and many other areas of concern, the impacts of uncertainty and volatility on the stock market are one of the most investigated issues. 
Uncertainty has the capacity to influence investment, hiring, consumption, financing costs, asset prices, output growth, and other economic outcomes in theory. Economic policy uncertainty, according to Tsai (2017), has a major impact on the Chinese stock market and its contagion risk spreads to different regional markets, except for Europe, although the impact of economic policy uncertainty in the United States is smaller than in China. In G7 countries, Ahmad \& Sharma (2018) discovered substantial results between the output gap and economic uncertainty. Other research has found that global stock market uncertainty is more significant than global bond market uncertainty (Dimic, Kiviaho, Piljak, \& Äijö, 2016). Global economic policy uncertainty has had a substantial negative impact on stock markets in Malaysia (Hoque \& Zaidi, 2020) and the United States (Arouri et al., 2016) as well as on other commodities, such as WTI Crude Oil and a 100-ounce gold bar in the United States (Bams, Blanchard, Honarvar, \& Lehnert, 2017). On the contrary, research has shown that uncertainty has a big and positive impact on the Chinese stock market (Liu \& Zhang, 2015; Yang \& Jiang, 2016). Furthermore, Mora (2019) found that previous to 2010, both economic policy uncertainty and volatility had large impacts on stock market returns in Thailand, but these impacts were minimal after 2010 .

At the aggregated stock market, industry level, and firm level, global risk factors such as geopolitical risk, global economic policy uncertainty, and oil price have substantial impacts on stock performance (Antonakakis, Chatziantoniou, \& Filis, 2013; Antonakakis, Gupta, Kollias, \& Papadamou, 2017; Kang et al., 2017b; Kang et al., 2017a; Kang \& Ratti, 2013). Uncertainty over global economic policy is one of the most important global risk factors affecting global financial market performance (Arouri et al., 2016; Baker, Bloom, \& Davis, 2016). Following the Asian Financial Crisis, the 9/11 terrorist attacks, the US-led Iraq invasion in 2003, the Global Financial Crisis in 2008-09, the European immigration crisis, concerns about the Chinese economy in late 2015, and the Brexit referendum in June 2016, global economic policy uncertainty increased dramatically, according to Davis (2016). Davis (2016) created a global economic policy index based on the national economic policy indices of 16 nations that account for two-thirds of global output.

Economic uncertainty is also exacerbated by governments and political processes. Major economic shocks and disruptions can also result in policy-related uncertainty. The Global Financial Crisis of 2008-09 presented policymakers with unprecedented and challenging issues. There was a great deal of ambiguity about how authorities should and would respond to the problems, as well as the economic and financial implications, which include investment, hiring, consumption, financing costs, asset prices, output growth, and other economic outcomes (Fernández-Villaverde, Guerrón-Quintana, Kuester, \& Rubio-Ramírez, 2015).

Forecasts of monthly volatilities for the aggregate global gold markets are frequently based on global economic policy uncertainties (Fang, Chen, Yu, \& Qian, 2018). Using the Markov switching model, Yu \& Song (2018) explored the impact of global policy uncertainty on aggregate monthly volatility and discovered that global policy uncertainty can lead to significant fluctuations and higher projections. Fang et al. (2018) used the GARCH-MIDAS model to investigate the impact of global policy uncertainty on future gold return variance and found that GEPU can accurately estimate monthly volatility in gold markets. Li, Ma, Zhang, \& Zhang (2020) investigated the impact of global economic policy uncertainty on Chinese stock market volatility as well as the implications of directional policy uncertainty based on the shifting directions of global and Chinese policy uncertainty.

Interestingly, when the level of global economic policy uncertainty is taken into account, Bitcoin's hedging efficacy for both global equities and global bonds improves marginally (Fang, Bouri, Gupta, \& Roubaud, 2019). The volatility of the Chinese stock market has been found to be influenced by global economic policy uncertainties. Global economic policy uncertainty benefits financial and consumer discretionary businesses, but it hurts the information technology, materials, telecommunication services, and energy sectors, according to the report. In contrast to the mixed influence of global economic policy uncertainty in long-run volatility, long-run correlations across industries are all positively related to global economic policy uncertainty (Yu, Fang, \& Sun, 2018). 
According to Hoque \& Zaidi (2019), the effects of global economic policy uncertainty differ according to regime states, sectors, and the form of effects, with negative effects outnumbering good effects. In a high-volatility environment, global economic policy uncertainty has a greater impact on stock returns. As a result, the data support the existence of asymmetric, non-linear, non-monotonic, and state-dependent correlations between global economic policy uncertainty and Malaysian sectoral stock returns. Furthermore, Cepni, Guney, \& Swanson (2020) show strong evidence that utilising targeted predictors in conjunction with global uncertainty indices and global surprise indices based on professional forecasters' expectations leads to more accurate GDP forecasts for emerging market nations.

The expectation of price swings is captured by volatility, which is used to evaluate market mood among investors and traders. Traders and analysts use a range of indicators to measure volatility and decide the best time to exit or enter trades. While greater panic during significant market changes can offer purchasing opportunities and offer an lucrative trading ground for experienced investors, high volatility is typically a deterrent for a risky investment. Low volatility combined with investor complacency, on the other hand, may suggest frothy market conditions and probable market instability. The CBOE Volatility Index (VIX), which provides a real-time estimate of 30-day projected volatility, is one of the most widely used instruments for determining relative levels of volatility. The VIX index is calculated using a panel of options prices and represents the "risk-neutral" predicted stock market variance for the US S\&P500 contract (Wang, 2019). It's known as a "fear index", and it represents stock market jitters. Several research have looked into the relationship between the VIX and the volatility of stock market performance. Bekaert \& Hoerova (2014), for example, decomposed the squared VIX index, which is derived from US S\&P500 options prices, into conditional variance of stock returns and the equity variance premium. The association between changes in the fear index and changes in emerging market volatility was investigated by Badshah, Bekiros, Lucey, \& Uddin (2018) (namely Chinese, Brazilian, and the overall emerging volatility index). Mei, Zeng, Zhang, \& Hou (2018) looked into whether US economic policy uncertainty might provide useful predictive information for European stock market forecasting.

Following the COVID-19 pandemic, research has switched to investigating the pandemic's impact on stock markets, such as the Chinese stock market (Al-Awadhi, Alsaifi, Al-Awadhi, \& Alhammadi, 2020) and the world stock market (Hassan \& Gavilanes, 2021). Studies focusing on Islamic stock markets are still relatively underexplored, given this new trend. With the rise of Islamic finance, there is a pressing need to understand how global risk factors influence stock returns in these markets, presenting an opportunity for further research. By researching the effects of economic policy uncertainty and volatility on the Islamic market, we may contribute to this developing research topic by providing important insights for contagion and spill-over effect studies in other Islamic nations and areas.

\section{DATA AND METHODOLOGY}

This study aims to examine the effects of global economic policy uncertainty (GEPU) and volatility (VIX) on Islamic stock market return. GEPU is one of the most widely used global risk indicators to gauge the impact of uncertainty on the global financial market performance (Arouri et al., 2016; Baker et al., 2016). As a GDP-weighted average of the national economic policy uncertainty indices of 16 countries, GEPU can reflect the global condition of the economy in general, uncertainty, and policy-related matters. As such, GEPU is an appropriate measure of global policy uncertainty. We use monthly data from 2011:M5 to 2021:M5. The availability of the monthly global economic policy uncertainty data facilitates the frequency choice for this study. The sample range is dictated by availability of data on the stock indices extracted from Datastream. The ten selected Islamic stock indices are: (i) Dow Jones Islamic Market World Index, (ii) Dow Jones Islamic Market Malaysia Titans 25 Index, (iii) Jakarta SE Composite Index, (iv) S\&P Saudi Arabia Shariah Index, (v) FTSE NASDAQ Dubai Kuwait 15 Shariah Index, (vi) FTSE NASDAQ Dubai UAE 20 Index, (vii) FTSE NASDAQ Dubai Qatar 10 Shariah Index, (viii) FTSE Turkey 
Shariah Index, (ix) Bahrain Islamic Index, and (x) FTSE Pakistan Shariah Index. These stock indices were chosen because they constitute the largest volume in daily transactions and are the most active Islamic stock markets. Stock market prices are transformed as below to generate stock market returns series. All series are converted into natural logarithmic series in Equation 1.

$$
R_{i, t}=\operatorname{In}\left(\frac{P_{t}}{P_{t-1}}\right) \times 100
$$

where $R_{t}$ denotes monthly stock returns of the industry for time $t . P_{t}$ and $P_{t-1}$ represent the spot price of the stock and the price of the previous stock month, respectively.

In this study, we rely on the wavelet transform, which is a mathematical technique that can decompose a signal into multiple lower resolution levels by controlling the scaling and shifting factors of a single wavelet function (Torrence \& Compo, 1998). Coherence is one of the most widely used methods for measuring linear interactions. It is based on the Pearson correlation coefficient used in statistics but in frequency and time domain. It measures the mean resultant vector length or consistency of the cross-spectral density between two signals. Its squared value varies from 0 to 1 , indicating low and high linear frequential correlation. In this study, coherence is used as a reference standard for comparison with other methods.

\subsection{The Continuous Wavelet Transforms (CWT)}

According to Sharif, Aloui, \& Yarovaya (2020), the continuous wavelet transforms $N a(p, q)$ shows the projection of a wavelet $\psi($.$) in contrast to the time sequence a (t) \in K^{2}(\underline{R})$, which is calculated in Equation 2 :

$$
N_{a}(p, q)=\int_{-\infty}^{\infty} a(t) \frac{1}{\sqrt{q}} \Psi\left(\frac{t-p}{M}\right) d t
$$

An essential feature of this technique is its potential to decompose consequently and seamlessly recreate a time series a $(\mathrm{t}) \in K^{2}(\underline{\mathrm{R}})$ :

$$
a(t)=\frac{1}{\sigma_{\Psi}} \int_{0}^{\infty}\left[\int_{-\infty}^{\infty} N_{a}(p, q)_{\Psi_{p q}}(t) d u\right] \frac{d q}{M^{2}}, M>0
$$

Moreover, this technique preserves the power of the observed time sequence in Equation 3.

$$
\|a\|^{2}=\frac{1}{\sigma_{\Psi}} \int_{0}^{\infty}\left[\int_{-\infty}^{\infty}\left|N_{a}(p, q)\right|^{2} d p\right] \frac{d q}{M^{2}}
$$

In this study, we rely on the aforesaid flexible tactic in the form of the wavelet coherence, which enumerates the successiveness between two time series in a bivariate model (see Equation 4).

3.2. The Wavelet Coherence $(W C)$

The wavelet formulation shows coherence between two signals, $x$ and $y$, and in the frequency $w$ and $t$ time domain. It is developed to explore the multiscale coherence and phase properties of time-varying non-stationary 
processes. First, the cross wavelet power and cross wavelet transform (CWT) is defined. Torrence \& Compo (1998) stated that the cross wavelet transform can be clarified by two-time sequence $a(t)$ and $b(t)$ as:

$$
N_{a b}(p, q)=N_{a}(p, q) N_{b}^{*}(p, q)_{(5)}
$$

where $N a(p, q)$ and $N b(p, q)$ in Equation 5 denote two continuous transforms of $a(t)$ and $b(t)$, respectively, where $p$ is the location index, $q$ denotes the measure, and $\left(^{*}\right)$ denotes the composite conjugate. $|N a(p, q)|$ can be used to determine wavelet power using the cross wavelet transform. The cross wavelet power spectra distinguish the part of the domain associated to time-frequency compared to the time series under consideration in which substantial energy concentration is revealed (cumulus of the restricted variance). The wavelet coherence technique (WCT) can identify specific areas in the time-frequency domain where unexpected and significant differences in the co-movement patterns of the time series under study occur. According to Torrence \& Compo (1998), the coefficient of adjusted wavelet coherence has the following equation:

$$
W^{2}(p, q)=\frac{\left|M\left(M^{-1} N a b(p, q)\right)\right|^{2}}{M\left(M^{-1}|N a(p, q)|^{2}\right) M\left(M^{-1}|N b(p, q)|^{2}\right)}
$$

where $M$ is the smoothing mechanism, and $O \leq W 2(p, q) \leq 1$ shows the range of squared wavelet coherence coefficient. Closeness to zero is the indication of the absence of correlation, while closeness to unity is the indication of a high correlation. The Monte Carlo simulation method is used to examine the hypothetical allocation of wavelet coherence.

\section{RESULTS AND DISCUSSION}

The aim of this study is to examine the relationship between Islamic stock returns and global economic policy uncertainty (GEPU) and volatility (VIX). Figure 1 shows the plot of the time series trend of Islamic Stock Indices, GEPU, and the VIX for ten years from August 2011 to August 2021 with approximately 120 total observations. Based on Figure 1, the majority of the stock markets were adversely affected by the COVID-19 pandemic from late 2019 up to mid-2020 due to worldwide lockdowns. The downward trend reversed in mid-2020 when certain countries started to recover, and more money started going into the stock market. Some industries were more affected than others due to the lockdowns, hence postponing investment in those industries and redirecting the funds into the stock market. The impact, however, varies between markets, which is depicted in the degree of steepness in each stock market in Figure 1. DJIM, Turkey, and Kuwait seem to have had a brief downward plunge during that period. As expected, the pandemic increased uncertainty and volatility, which is shown by both GEPU and the VIX during this period. After mid-2020, these markets begin their bullish upward trend as economic recovery kicks in and investors' confidence returns.

Continuous wavelet transform (CWT) plots for each stock return and GEPU are shown in Figure 2. The movements of each variable in time scales and frequency bands are described by CWT. The horizontal axis depicts time, which ranges from 2011:M5 to 2021:M5, while the vertical axis depicts frequency, with 0-4 denoting short term, 4-8 denoting medium duration, 8-16 denoting long term, and 16-32 denoting extremely long term. The wavelet coherence identifies regions in time-frequency space where the two-time series co-vary. Warmer colours (yellow) indicate places with significant interconnectedness, while cooler colours (blue) indicate lower interaction. Beyond the important areas, there are cold zones that reflect time and frequencies with no dependency in the series. The lead/lag phase relationships between the studied series are shown by an arrow in the wavelet coherence plots. On a given scale, a zero-phase difference means that the two-time series move in lockstep. When the time series are in phase, the arrows point to the right (left) (anti-phase). When two series are in phase, the variables move in the same way; when the two series are anti-phase, the variables move in the opposite direction. The first variable is 
leading, with arrows pointing down to the right or up to the left, whereas the second variable is leading, with arrows pointing up to the right or down to the left.

Figure 2 shows the results of the continuous wavelet power spectrum for Islamic stock returns and GEPU. It can be easily seen that in the case of GEPU, there yellow regions in small, medium, and long periods. As for the returns, a yellow region is visible on small and medium term but lacks in the long term. Significant interrelation for GEPU mostly exists in the medium term during 2015-2017 and 2019-2021 and also for long term in between the 8-16 period. However, in the very long term, between 16-32, there is a less significant interrelation for these two variables.

In the case of the DJIM, we identify a huge island which shows that strong dependency exists in medium term periods (4-8) during 2011 and from 2018 to 2020. The August 2011 stock market crash affected stock exchanges in the US, Middle East, Europe, and Asia. Sharp drops in these exchange markets emanated from the lower credit ratings due to concerns over the sluggish US economic growth and the fear of the possible contagion effect of the European sovereign debt crises in Spain and Italy. In 2018, major US stock indices had their worst annual performance since the beginning of the financial crisis; the Dow Jones Industrial Average dropped, and a similar blow was experienced by the DJIM. In 2020, the stock market plummeted, which signalled the beginning of the COVID-19 recession. However, from 2013 to 2017, the areas of blue colour are quite stable for all small, medium, and long periods. This suggests that the stock market was performing well during this period. It is interesting to observe that in cross wavelet transform, the path of arrows at various frequency bands point in a similar direction. This indicates that the returns for the DJIM and GEPU have a positive significant interrelation because the arrows point to the right when the time series are in phase. When the two series are in phase, this indicates that the variables move in the same direction where arrows pointing down to the right show that the second variable is leading. In this case, GEPU is leading from the stock return. Similarly, Kuwait and Qatar also have a positive significant interrelation, but, in this case, the first variable, stock return, is leading because of the arrows pointing up to the right compared to the DJIM where the arrow is pointing down to the right.

Other stock market returns, such as Turkey, Pakistan, the UAE, Bahrain, Indonesia, and Malaysia, clearly show that the arrows point to the left when the time series are in anti-phase. This shows that the stock returns of these countries have a negative significant interrelation in strong dependence regions. For Turkey, Pakistan, the UAE, and Indonesia, GEPU is the leading variable. On the contrary, in the case of Bahrain, Indonesia, and Malaysia stock return is the leading variable. Saudi Arabia provides an interesting case. The variant region is small, but the direction of the arrow is mixed. In 2011 and 2019, the arrows show a negative relationship where GEPU is the leading variable, but in 2013 and 2015 they show a positive and significant relationship where GEPU is the leading variable. Overall, the power dynamics of most of the regions in the cross wavelet power spectrum are moderately high. In general, we can conclude that the cross wavelet suggests a link between stock return and GEPU. Our results contradict the findings of Aziz, Marwat, Mustafa, \& Kumar (2020), who investigated the volatility spillovers from global economic policy uncertainty and macroeconomic factors to the Islamic stock market returns. The results suggest that the Islamic stock markets are less likely to be influenced by global economic policies and macroeconomic factors. However, this study echoes the findings by Hoque \& Zaidi (2019) for Malaysia's stock market where global economic policy uncertainty and oil demand shock factors affect stock market returns.

Figure 3 shows CWT plots for stock market returns and the volatility index (VIX). We identified an island of high dependence in the long term, that is $8-16$, over the entire sample period, and the arrows are mostly pointing up to the left, inferring a significant negative interrelation between stock return and the VIX with the VIX as the leading variable. Small islands of strong dependence were detected at the beginning of 2013 and the island began to increase in size from 2017 until now. This may reflect "investor fear gauge" where the VIX is often seen as a reflection of investor perception of the level of risk, or concern in the broader equities market. The 'fear' may have accumulated over the years, for example, during the financial crises in 1987 (Black Monday), 1997 (Asian Financial 
Crisis) and 2007 (US Subprime Crisis). These crises show a particular pattern on how the markets move in cycles of ten years. If investors were to believe in the 10-year cycle, by 2017, investors would be expecting another financial downturn where the slightest trigger could have led to a stock market crash. In 2020, the stock market downturn was a worldwide phenomenon due the COVID-19 pandemic, which led to economic recession. These collective fears were captured by the VIX. Our results show that the VIX negatively affects the DJIM stock returns.

In the case of Turkey, small regions of yellow were detected in the medium term period (4-8 months) in 2013 to 2017. Similarly, Bahrain exhibited a small region of yellow for a short-term period in 2012. Qatar also has a small island of yellow in the short and medium terms, but the arrow pointing in phase shows that they have a significant positive relationship, and it points upward in 2019 indicating that the VIX is the leading variable. In Malaysia, a very weak dependency between the VIX and returns was detected in the medium term and none in the short and long terms. The arrow is in phase and indicates a positive relationship between stock market returns and the VIX, and the arrow is pointing down to the right, which infers that stock return is the leading variable.

Pakistan's stock return also has a negative and significant relationship with the VIX but only in 2017 to 2020 for 8-16 months. In this case, the arrow is pointing up to the left, which shows that the VIX is leading. On a similar note, Indonesia's island is smaller than Pakistan's and the range is much shorter. The UAE and Kuwait have a negative significant relationship, with stock return being the leading variable. Bigger islands or coherence only appear in the very long term with a range between 16-32 from 2015 to 2017. Last, Saudi Arabia exhibits a bigger island, or stronger interrelation, in the short, medium, and long terms from 2011 to 2013 and from 2019 to 2020. The arrow is pointing down to the right and shows a positive relationship where VIX is leading for both periods, but in the short term, stock return is leading because the arrow is pointing up to the right.

The findings of this analysis are consistent with those of Aloui, Gupta, \& Miller (2016), who used a wavelet technique to show a significant level of correlation between investor sentiment and Islamic stock returns from 1990:M4 to 2010:M12. Perez-Liston, Huerta, \& Haq (2016) investigated whether investor sentiment influences the stock returns of the Dow Jones Islamic Index (the market, large-cap, medium-cap, and small-cap index) from 1996:M12 to 2012:M5, and they discovered a positive correlation between investor sentiment and the Shariahcompliant index's returns.
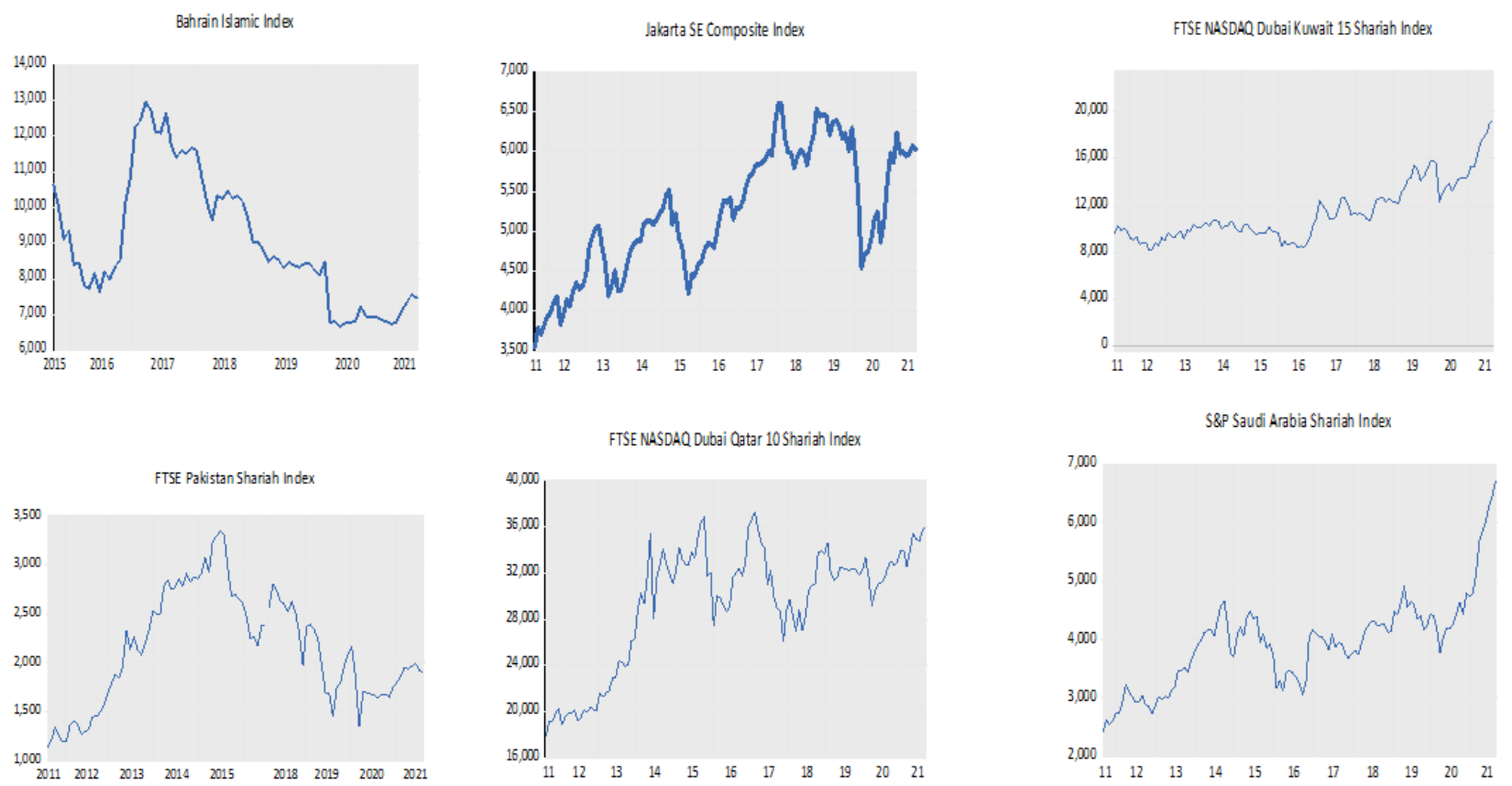

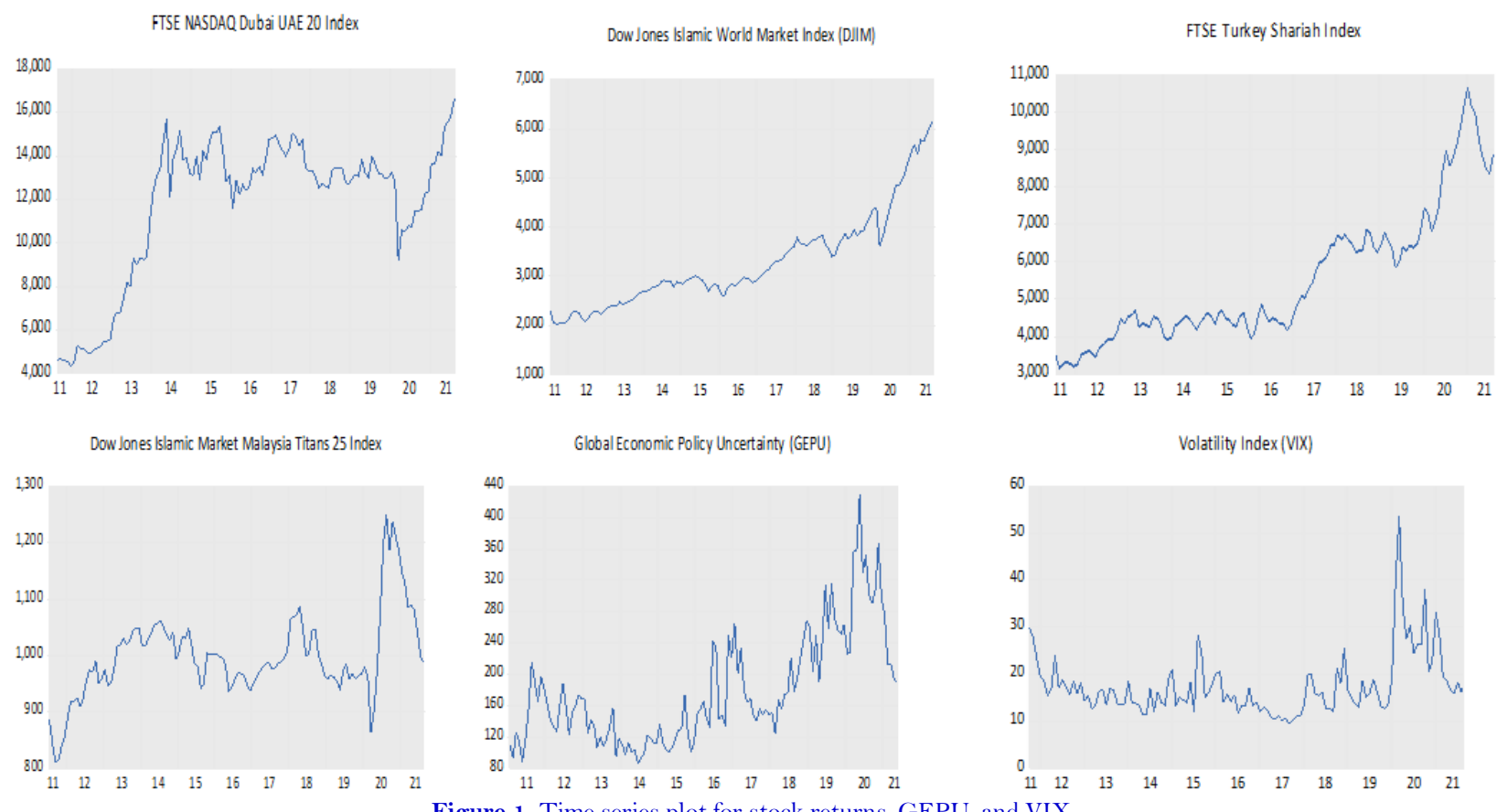

Figure 1. Time series plot for stock returns, GEPU, and VIX.
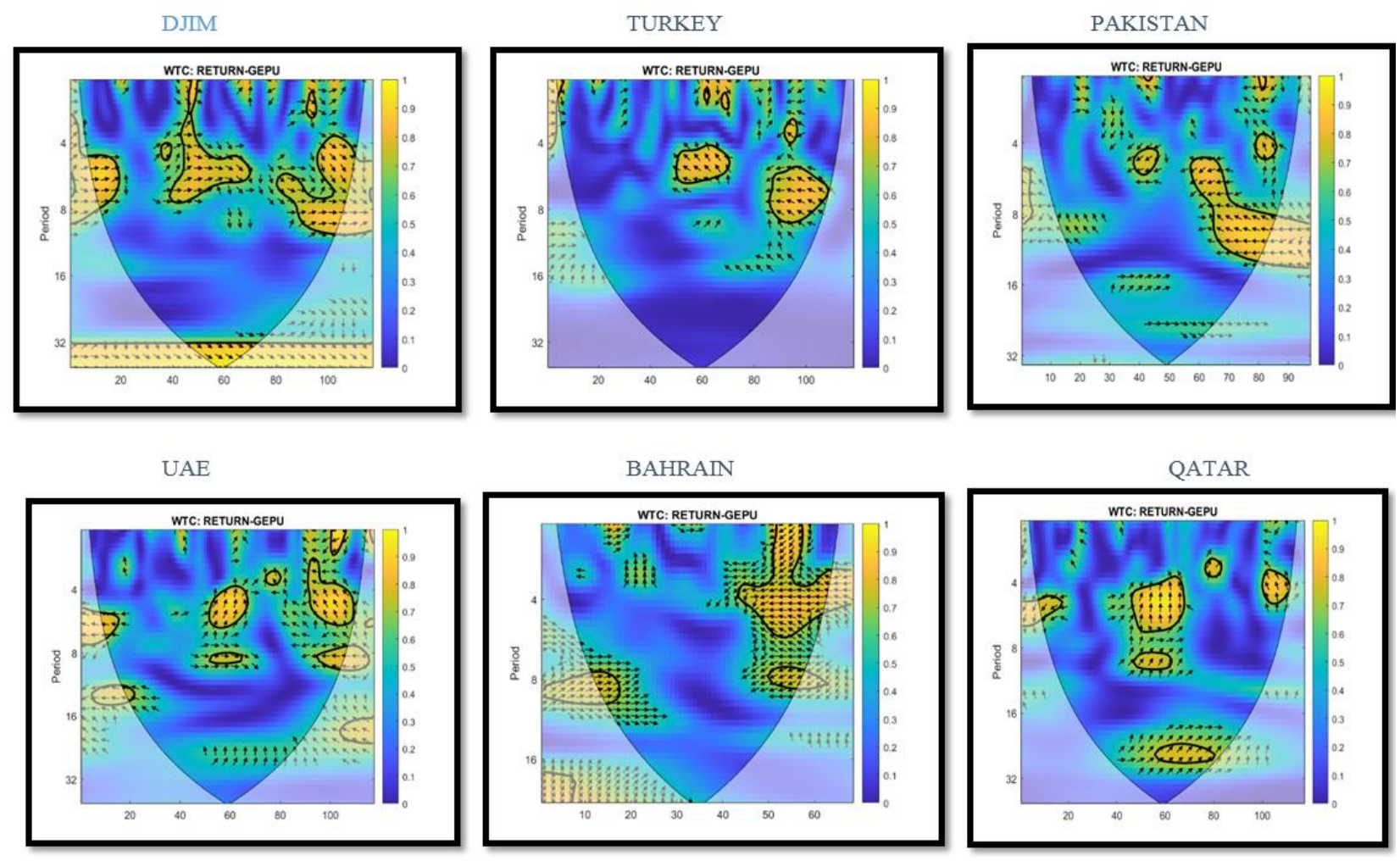
KUWAIT

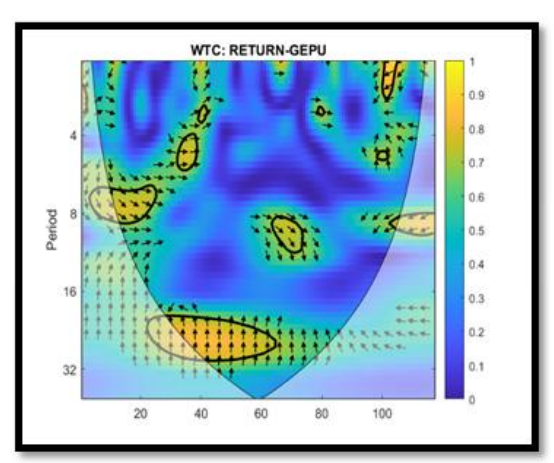

SAUDI ARABIA

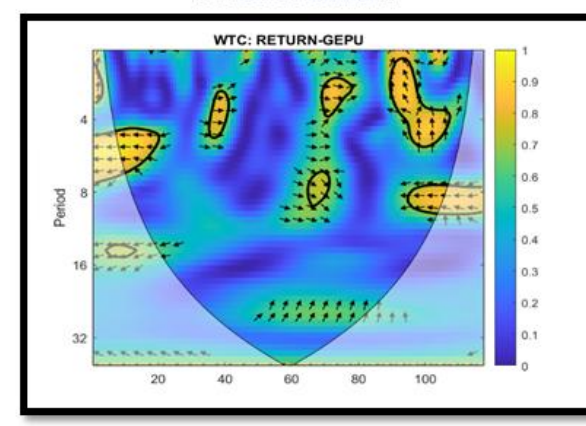

INDONESIA

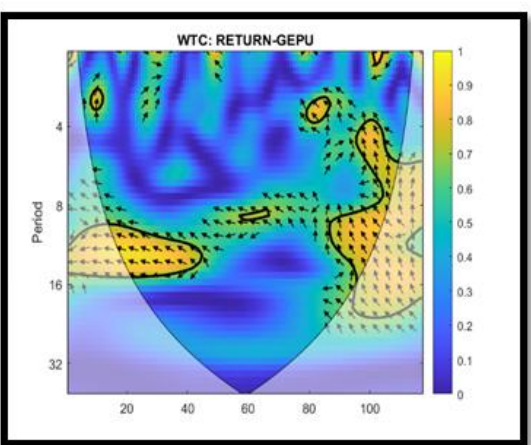

\begin{tabular}{|l|l|}
\hline 20 & $2011-2013$ \\
\hline 40 & $2013-2015$ \\
\hline 60 & $2015-2017$ \\
\hline 80 & $2017-2019$ \\
\hline 100 & $2019-2021$ \\
\hline
\end{tabular}

Figure 2. CWT plots for the stock market returns and global economic policy uncertainty (GEPU).

Note: The thick black contour indicates the 5\% significance level against yellow noise. The cone of influence specifies the region affected by edge effects and is displayed outside of the black line. The color code for power ranges from blue (low) to yellow (high). The phase difference between the two series is indicated by arrows. Arrows pointing to the right mean that the variables are in phase; arrows pointing to the left mean that the variables are out of phase. In phase indicates that variables have a cyclical effect, and out of phase or anti-phase means that the variables have an anti-cyclical effect on each other.
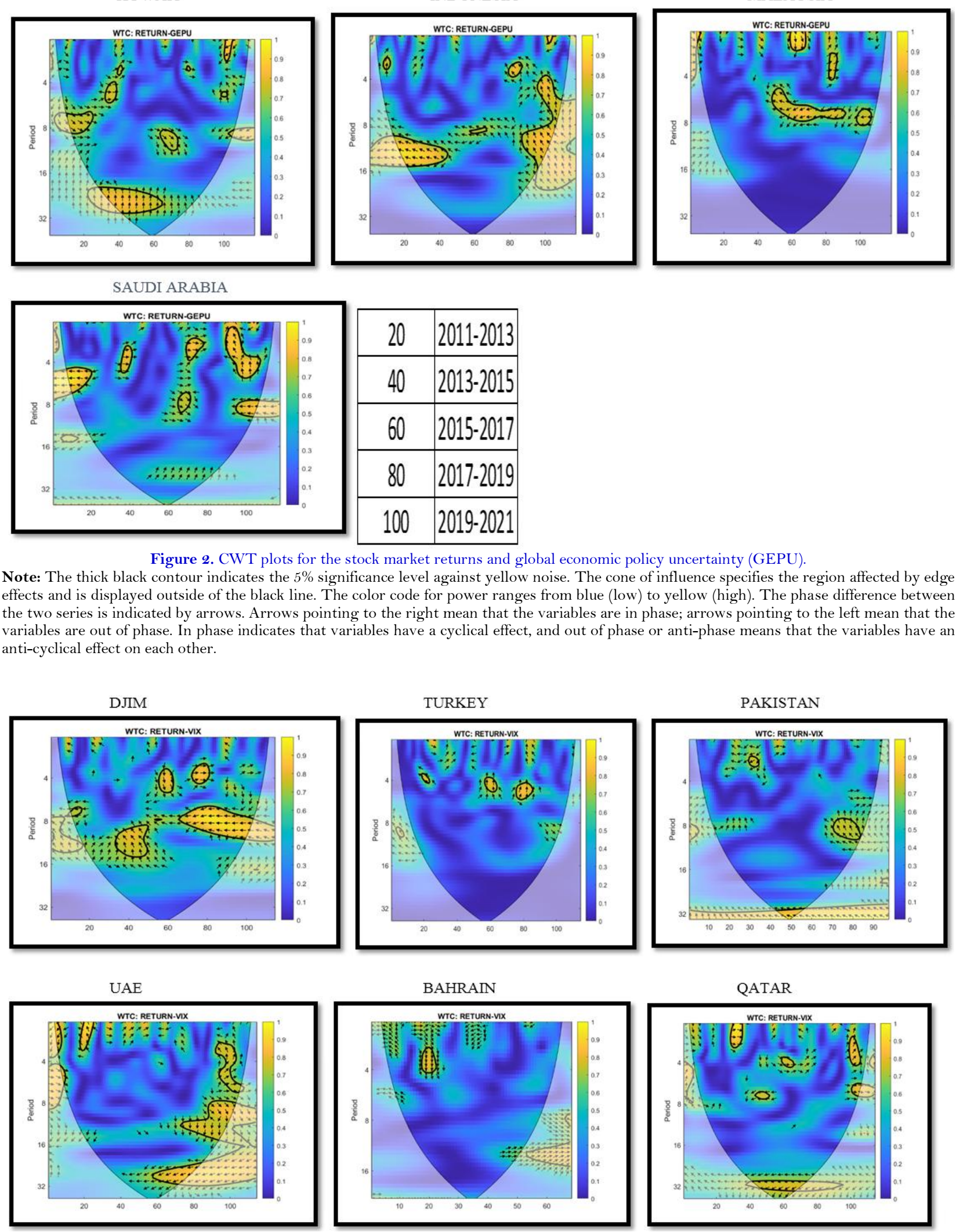
KUWAIT

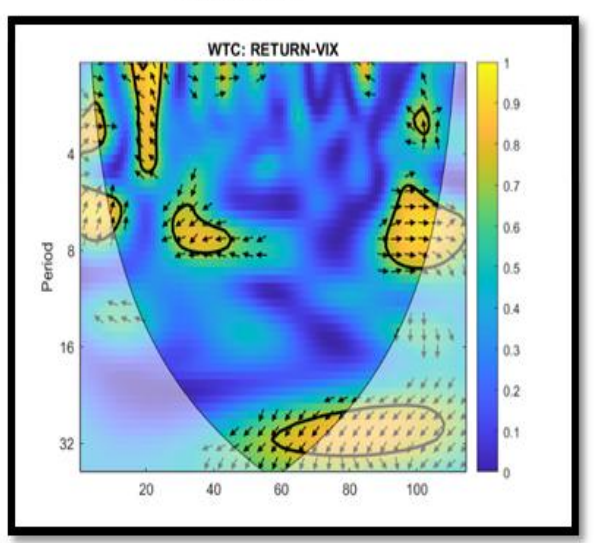

SAUDI ARABIA
INDONESIA

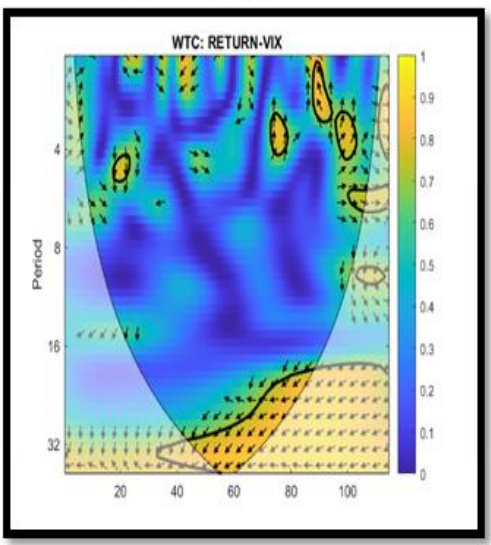

MALAYSIA

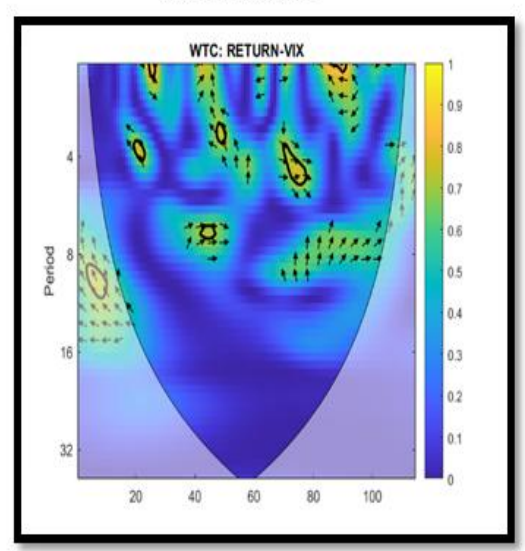

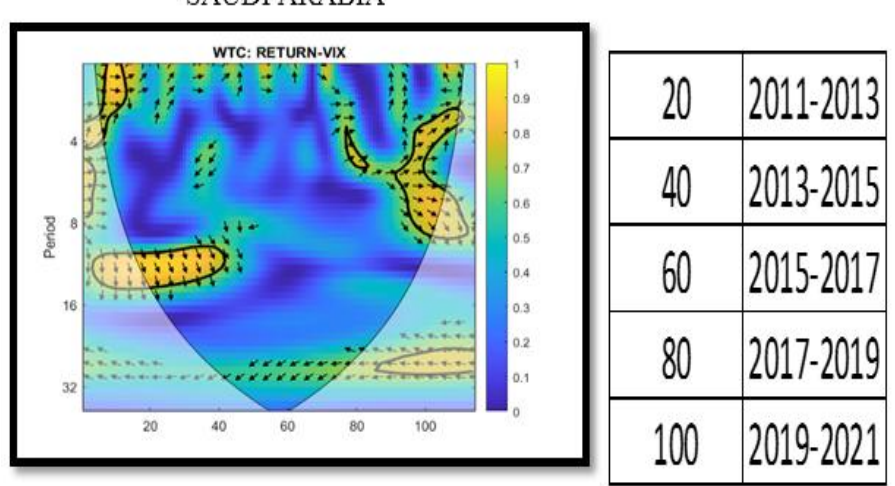

Figure 3. CWT plots for the stock market returns and the volatility index (VIX).

Note: The thick black contour indicates the $5 \%$ significance level against yellow noise. The cone of influence specifies the region affected by edge effects and is displayed outside of the black line. The color code for power ranges from blue (low) to yellow (high). The phase difference between the two series is indicated by arrows. Arrows pointing to the right mean that the variables are in phase; arrows pointing to the left mean that the variables are out of phase. In phase indicates that variables have a cyclical effect, and out of phase or anti-phase means that the variables have an anti-cyclical effect on each other.

\section{CONCLUSION}

In this study, we investigate the relationship between economic policy uncertainty and volatility proxied by GEPU and the VIX respectively on ten Islamic stock markets in the period from 2011:M5 to 2021:M5. A special focus is given to analysing the impact of these variables on the Islamic stock returns from a multi-scale perspective. Previous literature has focused primarily on the conventional stock markets, especially regarding macroeconomic variables. Since the relationship between GEPU and the VIX on Islamic stock returns is still underexplored, this study attempts to address this gap. In this study, we analyze the time-frequency connectedness between GEPU, VIX, and the Islamic stock market returns using the the wavelet coherence method.

The result shows that the impact of GEPU and VIX on Islamic stock returns may vary depending on the time scale. In the short and medium terms, the fluctuations of GEPU and the VIX have a significant relationship with the ten selected Islamic stock returns. In the case of global economic policy uncertainty, there is no major impact in the short run for all stock markets except Bahrain's. Significant impacts can be seen in the medium and long terms for all stock markets except Kuwait's. These significant regions fall in the extremely long-term period (16-32). In nine out of the ten stock markets studied, a significant and negative relationship was found between stock returns and GEPU; the DJIM was found to have a positive relationship.

The result for the VIX shows there is no significant relationship between both stock return and the VIX in the short-term period. The majority of the stock market returns under study have a positive relationship with the VIX. Most of the yellow regions fall in the medium- and long-term periods. Thus, our results provide several important pieces of evidence. First, GEPU had a greater effect on the Islamic stock market after the COVID-19 outbreak. This is shown by several huge hot yellow areas identified over the medium and long-term or 4-to-16-month frequency 
band beginning in 2020. Therefore, we recommend including these two variables in future analyses. Second, even though the impact of the VIX is no greater than GEPU, it is still an important factor to consider due to the longterm effect on the stock market as depicted in the DJIM and the UAE. Third, the outcomes of this study show the effectiveness of the wavelet approach in detecting the real underlying data features as the power spectrum testified the persistence and dominance of short-term drifts and spikes while the wavelet coherence enables us to adequately encircle all areas and periods of high positive co-movements. The main goal of this study is to improve the previous findings reported in recent and pioneering empirical works and provide credible results and fresh insights. Contrary to expectations, economic policy uncertainty and volatility may not necessarily always affect the stock market in a negative manner, as shown by our analysis. This provides opportunities for investors to reap the benefits in these markets. The results can be used by both policy makers and investors to reorient their strategies or rebalance their portfolios in view of the relative risks in these markets. Policy makers could redesign existing policies based on this new understanding of the interaction of stock market returns, economic policy uncertainty, and volatility to suit the current situation and to prevent potential market crashes in the future.

Funding: This research is funded by the Ministry of Higher Education Malaysia; Grant No.: FRGS/1/2019/SSO1/UITM/02/40.

Competing Interests: The authors declare that they have no competing interests.

Authors' Contributions: All authors contributed equally to the conception and design of the study.

\section{REFERENCES}

Ahmad, W., \& Sharma, S. K. (2018). Testing output gap and economic uncertainty as an explicator of stock market returns. Research in International Business and Finance, 45, 293-306. Available at: https://doi.org/10.1016/j.ribaf.2017.07.162.

Al-Awadhi, A. M., Alsaifi, K., Al-Awadhi, A., \& Alhammadi, S. (2020). Death and contagious infectious diseases: Impact of the COVID-19 virus on stock market returns. Journal of Behavioral and Experimental Finance, 27, 100326. Available at: https://doi.org/10.1016/j.jbef.2020.100326.

Aloui, R., Gupta, R., \& Miller, S. M. (2016). Uncertainty and crude oil returns. Energy Economics, 55, 92-100. Available at: https://doi.org/10.1016/j.eneco.2016.01.012.

Antonakakis, N., Chatziantoniou, I., \& Filis, G. (2013). Dynamic co-movements of stock market returns, implied volatility and policy uncertainty. Economics Letters, 120(1), 87-92. Available at: https://doi.org/10.1016/j.econlet.2013.04.004.

Antonakakis, N., Gupta, R., Kollias, C., \& Papadamou, S. (2017). Geopolitical risks and the oil-stock nexus over $1899-2016$. Finance Research Letters, 23, 165-173. Available at: https://doi.org/10.1016/j.frl.2017.07.017.

Arouri, M., Estay, C., Rault, C., \& Roubaud, D. (2016). Economic policy uncertainty and stock markets: Long-run evidence from the US. Finance Research Letters, 18, 136-141. Available at: https://doi.org/10.1016/j.frl.2016.04.011.

Aziz, T., Marwat, J., Mustafa, S., \& Kumar, V. (2020). Impact of economic policy uncertainty and macroeconomic factors on stock market volatility: Evidence from islamic indices. The Journal of Asian Finance, Economics, and Business, 7(12), 683-692. Available at: https://doi.org/10.13106/jafeb.2020.vol7.no12.683.

Badshah, I., Bekiros, S., Lucey, B. M., \& Uddin, G. S. (2018). Asymmetric linkages among the fear index and emerging market volatility indices. Emerging Markets Review, 37, 17-31. Available at: https://doi.org/10.1016/j.ememar.2018.03.002.

Baker, S. R., Bloom, N., \& Davis, S. J. (2016). Measuring economic policy uncertainty. The Quarterly Journal of Economics, 131(4), 1593-1636.

Bams, D., Blanchard, G., Honarvar, I., \& Lehnert, T. (2017). Does oil and gold price uncertainty matter for the stock market? Journal of Empirical Finance, 44, 270-285. Available at: https://doi.org/10.1016/j.jempfin.2017.07.003.

Bekaert, G., \& Hoerova, M. (2014). The VIX, the variance premium and stock market volatility. Journal of Econometrics, 183(2), 181-192. Available at: https://doi.org/10.1016/j.jeconom.2014.05.008. 
Cepni, O., Guney, I. E., \& Swanson, N. R. (2020). Forecasting and nowcasting emerging market GDP growth rates: The role of latent global economic policy uncertainty and macroeconomic data surprise factors. Journal of Forecasting, 39(1), 18-36. Available at: https://doi.org/10.1002/for.2602.

Davis, S. J. (2016). An index of global economic policy uncertainty. NBER Working Paper, No. (w22740).

Dimic, N., Kiviaho, J., Piljak, V., \& Äijö, J. (2016). Impact of financial market uncertainty and macroeconomic factors on stockbond correlation in emerging markets. Research in International Business and Finance, 36, 41-51. Available at: https://doi.org/10.1016/j.ribaf.2015.09.001.

Fang, L., Bouri, E., Gupta, R., \& Roubaud, D. (2019). Does global economic uncertainty matter for the volatility and hedging effectiveness of Bitcoin? International Review of Financial Analysis, 61, 29-36. Available at: https://doi.org/10.1016/j.irfa.2018.12.010.

Fang, L., Chen, B., Yu, H., \& Qian, Y. (2018). The importance of global economic policy uncertainty in predicting gold futures market volatility: A GARCH-MIDAS approach. Journal of Futures Markets, 38(3), 413-422. Available at: https://doi.org/10.1002/fut.21897.

Fernández-Villaverde, J., Guerrón-Quintana, P., Kuester, K., \& Rubio-Ramírez, J. (2015). Fiscal volatility shocks and economic activity. American Economic Review, 105(11), 3352-3384. Available at: https://doi.org/10.1257/aer.20121236.

Gulen, H., \& Ion, M. (2015). Policy uncertainty and corporate investment. The Review of Financial Studies, 29(3), 523-564.

Hassan, S., \& Gavilanes, J. M. R. (2021). First to react is the last to forgive: Evidence from the stock market impact of COVID 19. Journal of Risk and Financial Management, 14(1), 26. Available at: https://doi.org/10.3390/jrfm14010026.

Hoque, M. E., \& Zaidi, M. A. S. (2019). The impacts of global economic policy uncertainty on stock market returns in regime switching environment: Evidence from sectoral perspectives. International Journal of Finance \&ं Economics, 24(2), 9911016. Available at: https://doi.org/10.1002/ijfe.1702.

Hoque, M. E., \& Zaidi, M. A. S. (2020). Global and country-specific geopolitical risk uncertainty and stock return of fragile emerging economies. Borsa Istanbul Review, 2O(3), 197-213. Available at: https://doi.org/10.1016/j.bir.2020.05.001.

Kang, W., Gracia, F. P., \& Ratti, R. A. (2017b). Oil price shocks, policy uncertainty, and stock returns of oil and gas corporations. Journal of International Money and Finance, 70, 344-359. Available at: https://doi.org/10.1016/j.jimonfin.2016.10.003.

Kang, W., Ratti, R. A., \& Vespignani, J. L. (2017a). Oil price shocks and policy uncertainty: New evidence on the effects of U.S. and non-US oil production. Energy Economics, 66, 536-546. Available at: https://doi.org/10.1016/j.eneco.2017.01.027.

Kang, W., \& Ratti, R. A. (2013). Oil shocks, policy uncertainty and stock market return. Journal of International Financial Markets, Institutions and Money, 26, 305-318. Available at: https://doi.org/10.1016/j.intfin.2013.07.001.

Li, T., Ma, F., Zhang, X., \& Zhang, Y. (2020). Economic policy uncertainty and the Chinese stock market volatility: Novel evidence. Economic Modelling, 87, 24-33. Available at: https://doi.org/10.1016/j.econmod.2019.07.002.

Liu, L., \& Zhang, T. (2015). Economic policy uncertainty and stock market volatility. Finance Research Letters, 15, 99-105.

Mei, D., Zeng, Q., Zhang, Y., \& Hou, W. (2018). Does US economic policy uncertainty matter for European stock markets volatility? Physica A: Statistical Mechanics and its Applications, 512, 215-221. Available at: https://doi.org/10.1016/j.physa.2018.08.019.

Mora, A. (2019). The spillover effects of global economic policy uncertainty (GEPU) on emerging equity markets: Evidence from Thailand. Doctoral Dissertation, NIDA.

Perez-Liston, D., Huerta, D., \& Haq, S. (2016). Does investor sentiment impact the returns and volatility of Islamic equities? Journal of Economics and Finance, 4O(3), 42 1-437. Available at: https://doi.org/10.1007/s 12 197-014-9290-6.

Sharif, A., Aloui, C., \& Yarovaya, L. (2020). COVID-19 pandemic, oil prices, stock market, geopolitical risk and policy uncertainty nexus in the US economy: Fresh evidence from the wavelet-based approach. International Review of Financial Analysis, 70, 101496. Available at: https://doi.org/10.1016/j.irfa.2020.101496.

Torrence, C., \& Compo, G. P. (1998). A practical guide to wavelet analysis. Bulletin of the American Meteorological society, 79(1), 6178. 
Tsai, I.-C. (2017). The source of global stock market risk: A viewpoint of economic policy uncertainty. Economic Modelling, 60, 122-131. Available at: https://doi.org/10.1016/j.econmod.2016.09.002.

Wang, H. (2019). VIX and volatility forecasting: A new insight. Physica A: Statistical Mechanics and its Applications, $533,121951$.

Yang, M., \& Jiang, Z.-Q. (2016). The dynamic correlation between policy uncertainty and stock market returns in China. Physica A: Statistical Mechanics and its Applications, 461, 92-100. Available at: https://doi.org/10.1016/j.physa.2016.05.019.

Yu, M., \& Song, J. (2018). Volatility forecasting: Global economic policy uncertainty and regime switching. Physica A: Statistical Mechanics and its Applications, 511, 316-323. Available at: https://doi.org/10.1016/j.physa.2018.07.056.

Yu, H., Fang, L., \& Sun, W. (2018). Forecasting performance of global economic policy uncertainty for volatility of Chinese stock market. Physica A: Statistical Mechanics and its Applications, 505, 931-940. Available at: https://doi.org/10.1016/j.physa.2018.03.083.

Views and opinions expressed in this article are the views and opinions of the author(s), Asian Economic and Financial Review shall not be responsible or answerable for any loss, damage or liability etc. caused in relation to/arising out of the use of the content. 